\title{
Two Cases of Primary Malignant Fibrous Histiocytoma of the Liver: Immunohistochemical Expression of Ezrin and Its Relationship with Prognosis
}

\author{
Masahiko Sugitani', Osamu Aramaki ${ }^{1,2}$, Kentaro Kikuchi', Aleemuzzaman Sheikh', \\ Toshinori Oinuma ${ }^{1}$, Takao Mamiya ${ }^{2}$, Tadatoshi Takayama ${ }^{2}$ and Norimichi Nemoto ${ }^{1}$ \\ ${ }^{1}$ Department of Pathology, Nihon University School of Medicine and ${ }^{2}$ Department of Digestive Surgery, Nihon University \\ School of Medicine, Tokyo, Japan
}

Received January 13, 2009; accepted April 1, 2009; published online May 20, 2009

\begin{abstract}
Malignant fibrous histiocytoma (MFH) as soft tissue sarcoma would not be especially noteworthy, but primary hepatic MFH reports are extremely rare. Herein, we report ezrin expression in tumor tissues from two primary hepatic MFH cases with different prognoses. Cases 1 and 2 were both women, ages 45 and 70 years, respectively. Case 1 had an $11 \times 10 \mathrm{~cm}$ liver tumor in segment (S) 3, and case 2 had two liver tumors, $12 \times 8 \mathrm{~cm}$ in $S 5$ and $10 \times 7 \mathrm{~cm}$ in S8. Neither had any other systemic tumors. Cases 1 and 2 survived for two year and ten months and for eight and a half months, respectively, after the initial tumor resection. Microscopically, the tumors of these two cases were similar and showed proliferation of atypical cells, including spindle, pleomorphic and multi-nucleated giant cells arranged in storiform, sheet and/or fascicle patterns, with scattered foci of inflammatory cells, indicating MFH. Ezrin expression in tumor tissue from case 1 was sparse, whereas that of case 2 showed strong ezrin expression in many tumor cells. The present results indicate ezrin immunoreactivity in primary hepatic MFH to correlate possible with prognosis, which is consistent with reports on some other types of malignancies.
\end{abstract}

Key words: malignant fibrous histiocytoma, liver, ezrin, prognosis

\section{Introduction}

Malignant fibrous histiocytoma (MFH), first reported by O'Brien and Stout [17] in 1964, was initially considered to be rare. At a later time, MFH was recognized rather frequently among soft tissue tumors usually occurring in the extremities of middle-aged or elderly patients. However, the working group of World Health Organization for classification of tumors of soft tissue and bone met in 2002 [23], and suggested that the term of MFH was synonymous with undifferentiated pleomorphic sarcoma, which has become a diagnosis of exclusion accounting for less than $5 \%$ of adult sarcoma. MFH less commonly involves the abdomen and

Correspondence to: Masahiko Sugitani, M.D., Ph.D., Department of Pathology, Nihon University School of Medicine, 30-1 Oyaguchikami-machi, Itabashi-ku, Tokyo 173-8610, Japan.

E-mail: sugitani@med.nihon-u.ac.jp retroperitoneum, and Weiss and Enzinger [21] reported 16\% of MFH in a review of 200 cases to involve these sites. However, primary MFH of the liver is extremely rare. A review of 232 primary hepatic sarcomas, all histologically confirmed by the Armed Forces Institute of Pathology, from 1982 to 1990 , identified hepatic MFH in only 11 (4.74\%) [9]. MFH of the liver was first reported in 1985 [2, 5], and to date, only 35 cases have been described in the English literature, including recent reports by Anagnostopoulos et al. [3], Ding et al. [6], Ye et al. [24], Chen et al. [4] and Li et al. [13].

As for prognosis-related markers of malignant neoplasms in cytological and/or histological examinations, Akisawa et al. [1] reported high levels of ezrin expression by human pancreatic adenocarcinoma cell lines with high metastatic potential and Khanna et al. [10] suggested ezrin to possibly be a key factor in the metastasis of osteosarcoma, based on a microarray analysis. Ezrin is an 80 kilo- 
dalton protein and is present in placental microvilli, the microvillous surface of acid-secreting parietal cells of the gastric gland [20], membrane ruffles of cultured cells and growth cones of cultured neurons. The ezrin protein belongs to the ezrin-radixin-moesin (ERM) cytoskeleton-associated protein family, which are linkers between membrane proteins and actin filaments. The ezrin has a potentially important role in the cortical cytoskeleton, and may participate in the regulation of several cellular functions. Intensive investigations have shown strong ezrin immunoreactivity in tumor tissues to correlate with a poor prognosis in patients with malignancies, such as astrocytic tumors [8], melanoma [14], rhabdomyosarcoma [25], osteosarcoma [11], highly malignant soft tissue sarcoma [22], colorectal cancer [7] and squamous cell carcinoma of the head and neck [15]. However, positive and negative correlations between ezrin immunoreactivity and prognosis were found in ovarian carcinoma $[12,16]$ and pancreatic adenocarcinoma $[1,19]$. Based on these findings, ezrin expression is considered to promote metastasis and therefore to be a possible prognostic factor in some types of malignancies.

We experienced two cases with primary MFH of the liver in our university hospital. MFH of the liver is generally recognized as having a poor prognosis and both cases ultimately had the same outcome. However, one case died within a year while the other survived for nearly three years after tumor resection. We investigated ezrin expression using tumor sections from both cases. Only one report, to our knowledge, has described ezrin expression as an immunohistological prognostic factor in hepatic MFH [13]. This is because hepatic MFH itself is a rare tumor. Herein, we describe the possible correlation between clinical outcome and immunohistochemical expression of ezrin in primary hepatic MFH.

\section{Materials and Methods}

\section{Clinical courses of two cases}

The clinical course of Case 1, a 45-year-old Japanese woman, was as follows: She was followed up for a complaint of epigastralgia, at a nearby hospital for 4 months, and a hepatic mass was detected by computed tomography (CT) examination. It was located mainly in segment (S) 3. She was admitted to our hospital. A thorough systemic examination revealed no tumors anywhere else in the body. The patient underwent left hepatic resection under a preoperative diagnosis of possible intrahepatic cystadenocarcinoma. No tumors were found anywhere in the body, except that in the liver, intraoperatively. She was discharged from the hospital. However, hepatic recurrence was found in the remaining liver and partial resection of the right lobe was performed two years and five months later from the first operation. The absence of tumors anywhere in the body, other than the liver, was confirmed. Four months later from the second operation, surgery was again required. During the operation, a recurrent tumor invading the duodenum and pancreas was identified and gastro-jejunostomy was performed without tumor resection. Her final admission was due to abdominal fullness caused by an enlarged tumor and she died approximately two years and ten months after the initial resection. Autopsy revealed a recurrent MFH tumor that was located in the omental bursa and cause of death was considered to be due to sepsis.

The clinical course of Case 2, a 70-year-old Japanese woman, was as follows: She had undergone hysterectomy due to uterine prolapse at the age of 64 years. She was followed up under a diagnosis of possible pancreatitis at a neighborhood hospital one year prior to the admission described herein. Icterus and a liver tumor were recognized, and chemotherapy was administered at a local hospital in her hometown. She was referred for surgical treatment and was admitted to our hospital. On admission, two tumors were found in the right hepatic lobe on CT. A thorough systemic examination revealed no tumors anywhere else in the body. The liver tumors showed multi-cystic dilation of intrahepatic bile ducts, within the solid tumor masses. The patient underwent hepatic resection of S4, S5 and S8 under a preoperative diagnosis of intrahepatic bile duct cystadenocarcinoma. Postoperative recovery was uneventful and she was discharged from the hospital. However, recurrence in the liver and multiple lung metastases developed and she died approximately eight and a half months after the resection, at another hospital. Autopsy was not performed.

In both cases, anti-hepatitis $\mathrm{C}$ virus antibody and hepatitis B virus surface antigen were negative on serological examinations, levels of alpha-fetoprotein (AFP), carcinoembryonic antigen (CEA) and carbohydrate antigen 19-9 in serum were within normal limits, and preoperative needle biopsy examinations were not performed.

Thus, though their final outcomes were the same, the courses after tumor resection differed markedly between cases 1 and 2 . Informed consent was obtained from both cases.

\section{Histopathology and immunohistochemistry}

Histopathological and immunohistochemical examinations were performed on formalin-fixed and paraffinembedded tissue sections of the resected tumors. Immunohistochemical staining was performed to detect expressions of vimentin, alpha-1-antitrypsin, alpha-1-antichymotrypsin, CD68, S-100 protein, desmin, alpha-smooth muscle actin, myoglobin, myogenin, CD45 (leukocyte common antigen), CD30, Ki-67, epithelial membrane antigen, cytokeratin, AFP, CEA and ezrin. All antibodies used for pathological diagnosis except ezrin were from DakoCytomation (Glostrup, Denmark). Immunohistochemistry, except that for ezrin, employed the DAKO Autostainer according to the manufacturer's instructions. Monoclonal anti-ezrin antibody (clone 3C12) was obtained from Sigma-Aldrich (St. Louis, MO, USA). A positive control for ezrin immunostaining was used in the sections of placental microvilli. Based on a preinvestigation, sections from cases 1 and 2 were pretreated at $100^{\circ} \mathrm{C}$ with EDTA buffer at $\mathrm{pH} 8.5$ for $1 \mathrm{hr}$ and were incubated with 1:4000 diluted anti-ezrin antibody at $37^{\circ} \mathrm{C}$ for 1 
hr with an auto-immunostaining device (Ventana NX System, Ventana Medical Systems Inc, Tucson, AZ, USA), and all steps were carried out according to the manufacturer's instructions. Sections were observed microscopically (Olympus Corporation, Tokyo, Japan). The intensity and distribution of ezrin expression were estimated for both cases.

\section{Results}

Macroscopic findings of the liver in our two cases were as follows. The tumor from case 1 (Fig. 1) had a clear border measuring $11 \times 10 \mathrm{~cm}$ on the cut surface. The tumor was mostly solid, milky-white in color and partially cystic with necrosis and hemorrhage. The tumors from case 2 (Fig. 2) both had clear borders, with one measuring $12 \times 8 \mathrm{~cm}$, in proximity to $\mathrm{S} 5$, and the other $10 \times 7 \mathrm{~cm}$, in proximity to $\mathrm{S} 8$.

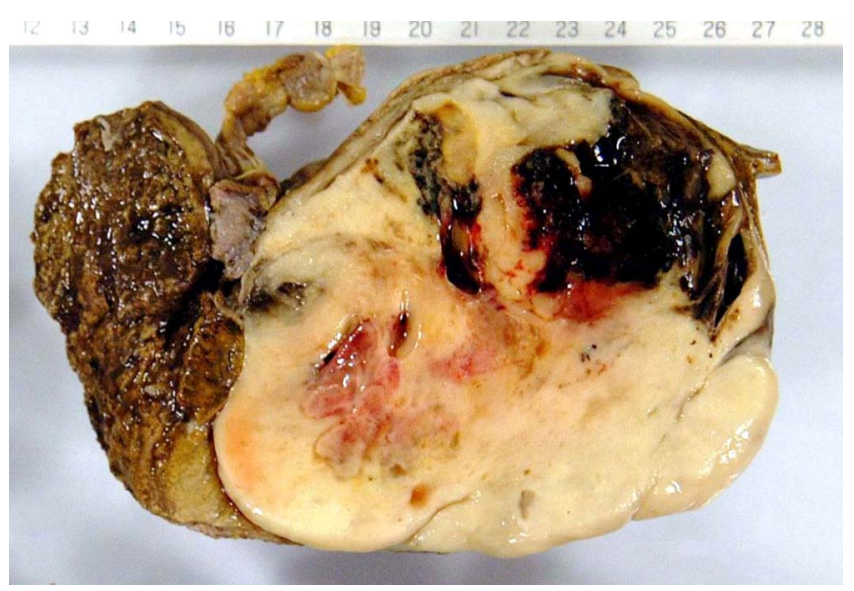

Fig. 1. Macroscopic findings of the cut surface of the tumor in case 1 .

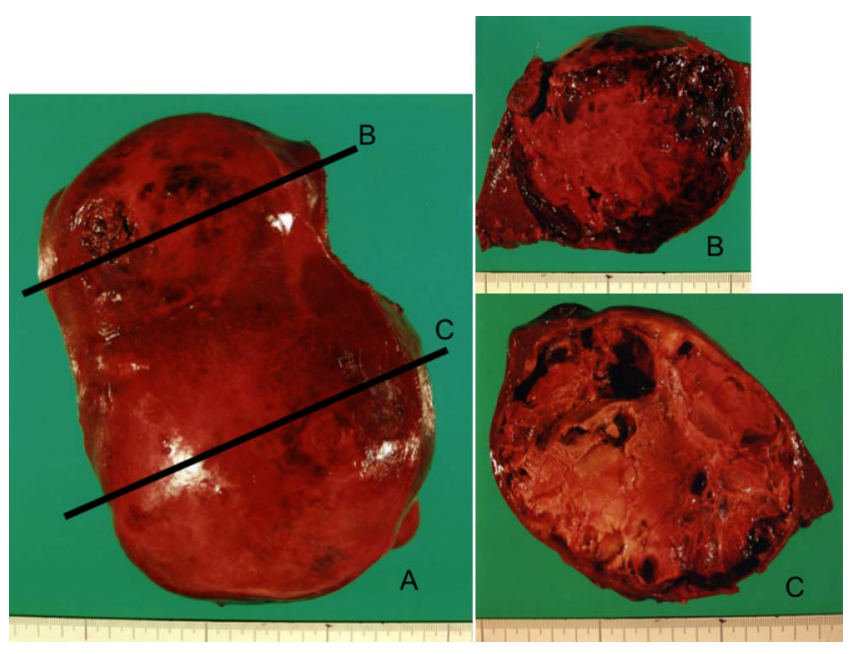

Fig. 2. Macroscopic tumor findings of the tumor in case 2. A; A large and a small tumor are present in the liver. Cut surfaces of the tumors are also shown (B and $\mathbf{C}$ ). The tumors were located mainly in segments 8 and 5 , respectively.
The cut surface showed both tumors to be yellowish-white in color. Necrosis was seen in both the S5 and the S8 tumor, and hemorrhage in the S8 tumor. The two tumors from case 2 were close but clearly not connected to each other.

Histopathological findings of the liver tumors from cases 1 (Fig. 3A and 3B) and 2 (Fig. 3C and 3D) were basically quite similar, and the two tumors from case 2 had nearly the same histopathological features. Microscopically, routine hematoxylin and eosin (H\&E) sections from the tumors showed proliferation of atypical cells composed of spindle, pleomorphic and multi-nucleated giant cells arranged in storiform, sheet and/or fascicle patterns containing areas of hemorrhage, coagulation necrosis and fibrosis. In both cases, nuclear atypia was equally prominent and mitoses were common (approximately 3-5 in 10 fields of high power view). Scattered foci of inflammatory cellular infiltration were also recognized. In comparison with the tumor from case 1 , those from case 2 had slightly higher cellularity and more frequent multi-nucleated giant cells. No osteoid, atypical adipocytes, rhabdoid cells or epithelial patterns were evident. The tumors showed infiltrative growth, but neither capsule nor fibrous septa formation within the tumor mass. The non-neoplastic areas had an essentially normal appearance, with no evidence of either chronic hepatitis or liver cirrhosis. Immunohistochemical examinations revealed the tumor cells to be positive for vimentin, alpha-1-antitrypsin, alpha-1-antichymotrypsin and CD68. The ratios of cells positive for Ki-67 to tumor cells were approximately $15 \%$ and $25 \%$ in cases 1 and 2 , respectively. There were no differences in staining patterns or stain affinities for these antibodies. Epithelial membrane antigen, cytokeratin, S-100 protein, desmin, alpha-smooth muscle actin, myoglobin, myogenin, CD45, CD30, AFP and CEA were all negative. The final pathological diagnosis of MFH was made for both cases.

Interestingly, the immunohistochemical detection of ezrin expression showed cytoplasmic or membranous staining but it differed markedly between cases 1 and 2 . In case 1 , the immunoreactivity against ezrin in tumor cells was weak and showed a scattered distribution (Fig. 4A). In contrast, in case 2 the staining was more intense and was diffusely observed in pleomorphic, mononuclear and/or multi-nucleated giant tumor cells (Fig. 4B and 4C). The ratios of cells positive for ezrin to tumor cells were less than $10 \%$ and approximately $40 \%$ in cases 1 and 2 , respectively.

\section{Discussion}

Two significant features are required to make a diagnosis of primary MFH of the liver. One involves the histopathology of the liver tumor. The morphological features of the tumor in our present cases were within the spectrum of soft tissue MFH, as described above. The other is that the clinicopathologic criteria for a primary hepatic origin must be met. Li et al. [13] proposed their criteria for primary hepatic MFH in a review of seven cases, as follows: solitary or multifocal liver neoplasm without evidence 

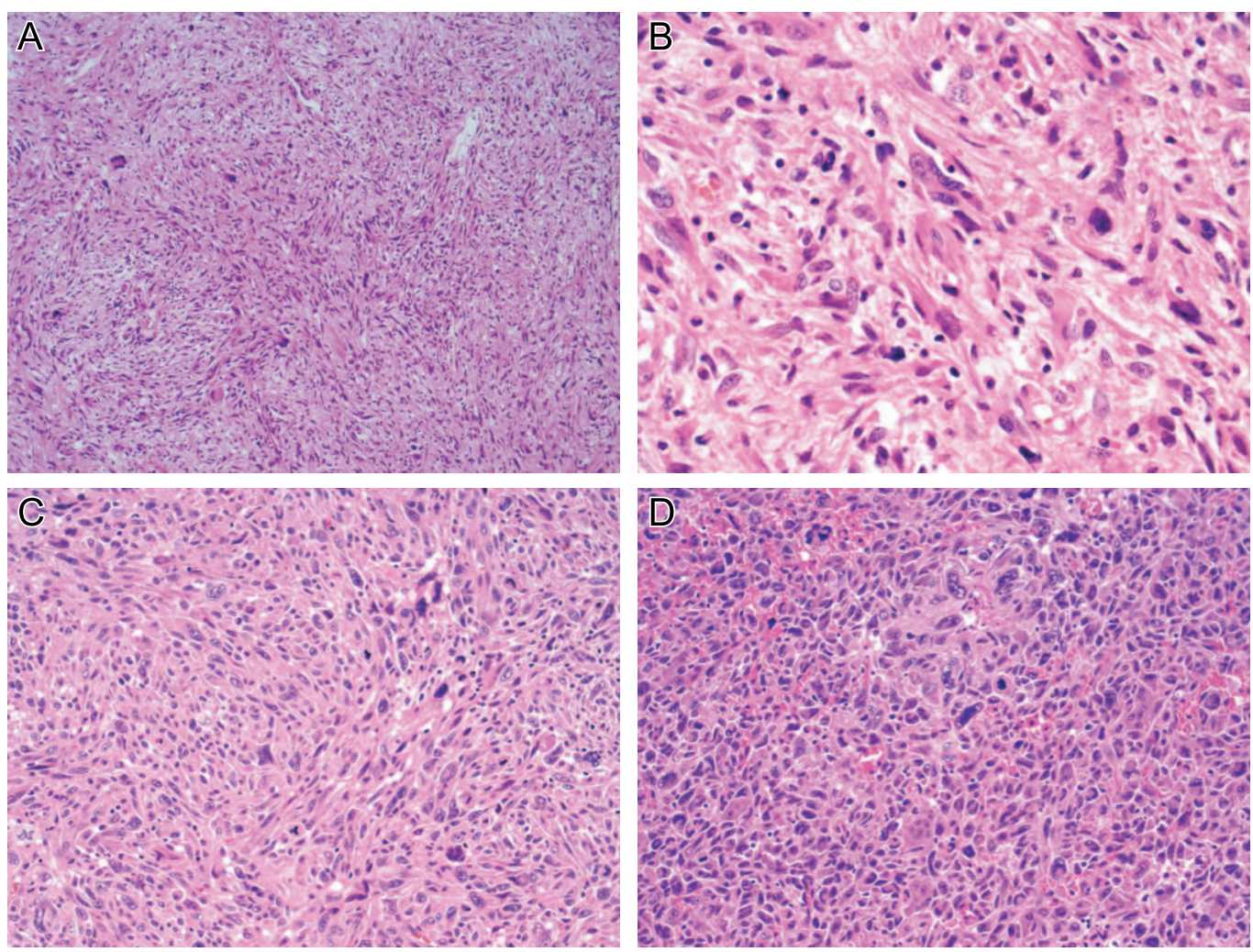

Fig. 3. Microscopic features of the MFH tumors in cases $1(\mathbf{A}, \mathbf{B})$ and $2(\mathbf{C}, \mathbf{D})$. They are quite similar. The storiform-pleomorphic patterns seen in MFH cases 1 and 2 are shown in $\mathbf{A}$ and $\mathbf{C}$, respectively. Proliferation of spindle, bizarre, pleomorphic and multi-nucleated giant cells in MFH cases 1 and 2 are shown in $\mathbf{B}$ and $\mathbf{D}$, respectively. In comparison with case 1, the tumor from case 2 had slightly higher cellularity and more frequent multi-nucleated giant cells. (H\&E staining, original magnification $\mathbf{A}, \times 100 ; \mathbf{B}, \times 400 ; \mathbf{C}$ and $\mathbf{D}, \times 200$ ).
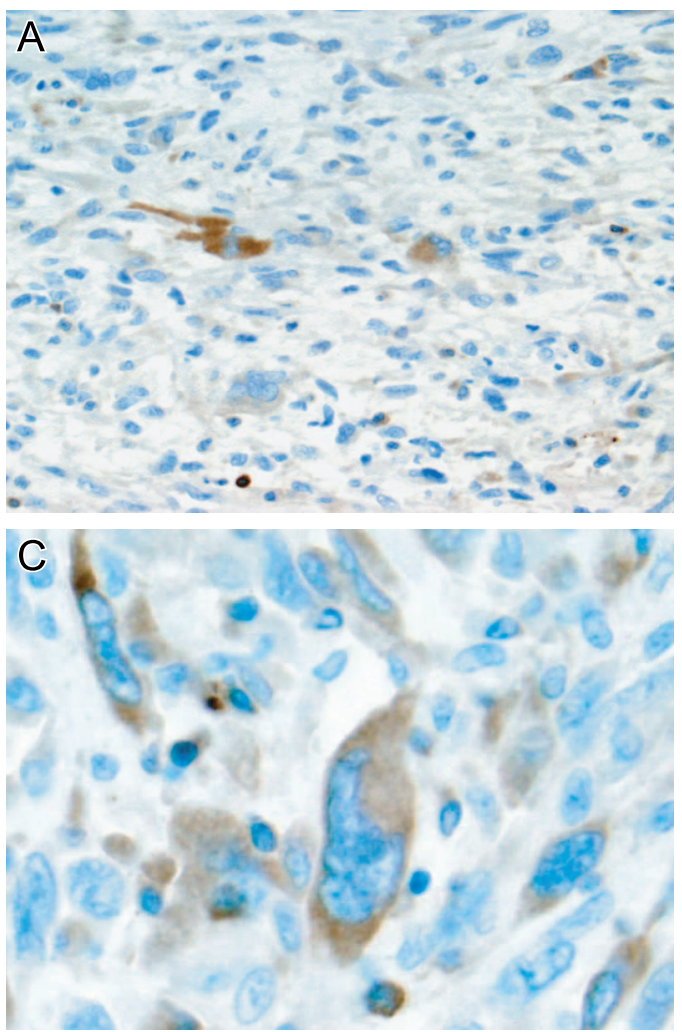

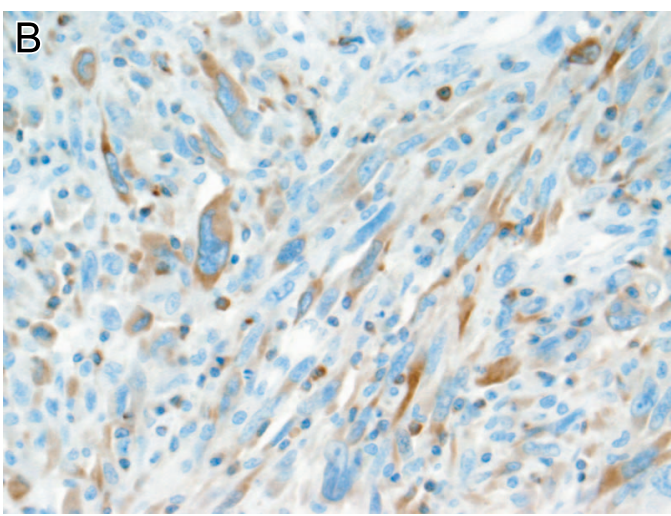

Fig. 4. Ezrin expressions in tumor cells from case 1 (A) and case 2 (B and $\mathbf{C})$. $\mathbf{C}$ is high power view of $\mathbf{B}$. Immunostaining with hematoxylin counter-staining is shown; original magnification of $\mathbf{A}$ and $\mathbf{B}$ is $\times 400$, and that of $\mathbf{C}$ is $\times 1000$. 
of a preexisting, concurrent, or subsequently discovered primary lesion in any part of the body. The most important point, in concluding that the origin is hepatic, is to exclude metastasis and direct invasion of $\mathrm{MFH}$ arising in other sites. In our cases, no tumors were found in any other organs or sites on radio-imaging examinations or intraoperative gross examination at the initial operation. These two cases showed features compatible with the criteria for primary MFH of the liver. In most hepatic MFH cases described in the literature [13], the tumor showed positive immunostaining for vimentin (8/9 cases), alpha-1-antitrypsin (6/7), alpha-1-antichymotrypsin (7/7) and CD68 (2/2), while being negative for cytokeratin (10/10), S-100 protein (9/9) and desmin (9/9). Our cases had the same immunohistochemical findings, allowing exclusion of myogenic and lipoblastic malignancies and sarcomatoid carcinoma. The immunohistochemical findings of the present cases were thus consistent with MFH.

The prognosis of a patient with soft tissue MFH depends greatly on tumor size, location and the adequacy of the initial surgical excision [18]. Tumor size is considered to be a clinically significant prognostic factor in hepatic MFH. On the other hand, histopathological grade is thought to actually be closely related to prognosis. In a previous study, when ezrin and other ERM family proteins were activated, they interacted with membrane proteins and the actin cytoskeleton, and as a result, they affected such processes as migration, adhesion, invasion and survival of the cell. These processes are important for the establishment and progression of malignant neoplasm. Therefore, the ezrin expression could be considered in relation with prognosis in several types of malignancies, including MFH. In the present study, the immunohistochemical expressions of ezrin clearly differed between cases 1 and 2. Clinically, they showed markedly different survival durations from the initial tumor resection. Recently, Li et al. [13] reported that three patients with high ezrin immunoreactivity died of their tumors within one year while two others with low ezrin immunoreactivity survived for more than four years after surgery. Their results indicated tumors with high immunohistochemical ezrin expression to be associated with a poor prognosis, whereas low expression was associated with a rather good prognosis. Although primary hepatic MFH is extremely rare, we could confirm the relationship between ezrin expression and prognosis, obtaining results similar to those reported by Li et al.

In conclusion, we have presented herein the immunohistochemical expression patterns of ezrin in two primary hepatic MFH cases. In determining the prognosis of patients with primary hepatic $\mathrm{MFH}$, as this disease is extremely rare, use of the histological grade of H\&E stained sections only is a limitation. Immunohistochemical ezrin staining was found to be useful as a supplementary method for determining prognosis. This study verified the immunohistochemical results reported by Li et al. [13], which showed a possible correlation between immunohistochemical ezrin expression and the outcomes of patients with primary hepatic MFH.

\section{Acknowledgment}

We wish to thank Dr. Kouji Hashimoto, Department of Surgery, Shimane Prefectural Central Hospital, for supporting this study.

\section{References}

1. Akisawa, N., Nishimori, I., Iwamura, T., Onishi, S. and Hollingsworth, M. A. (1999) High levels of ezrin expressed by human pancreatic adenocarcinoma cell lines with high metastatic potential. Biochem. Biophys. Res. Commun. 258; 395-400.

2. Alberti-Flor, J. J., O'Hara, M. F., Weaver, F., Evans, J., McClure, R. and Dunn, G. D. (1985) Malignant fibrous histiocytoma of the liver. Gastroenterology 89; 890-893.

3. Anagnostopoulos, G., Sakorafas, G. H., Grigoriadis, K. and Kostopoulos, P. (2005) Malignant fibrous histiocytoma of the liver: a case report and review of the literature. Mt. Sinai J. Med. $72 ; 50-52$.

4. Chen, H. C., Chen, C. J., Jeng, C. M. and Yang, C. M. (2007) Malignant fibrous histiocytoma presenting as hemoperitoneum mimicking hepatocellular carcinoma rupture. World J. Gastroenterol. 13; 6441-6443.

5. Conran, R. M. and Stocker, J. T. (1985) Malignant fibrous histiocytoma of the liver-a case report. Am. J. Gastroenterol. 80; 813815.

6. Ding, G. H., Wu, M. C., Yang, J. H., Cheng, S. Q., Li, N., Liu, K., Dai, B. H. and Cong, W. M. (2006) Primary hepatic malignant fibrous histiocytoma mimicking cystadenocarcinoma: a case report. Hepatobiliary Pancreat. Dis. Int. 5; 620-623.

7. Elzagheid, A., Korkeila, E., Bendardaf, R., Buhmeida, A., Heikkilä, S., Vaheri, A., Syrjänen, K., Pyrhönen, S. and Carpén, O. (2008) Intense cytoplasmic ezrin immunoreactivity predicts poor survival in colorectal cancer. Hum. Pathol. 39; 1737-1743.

8. Geiger, K. D., Stoldt, P., Schlote, W. and Derouiche, A. (2000) Ezrin immunoreactivity is associated with increasing malignancy of astrocytic tumors but is absent in oligodendrogliomas. Am. $J$. Pathol. 157; 1785-1793.

9. Ishak, K. G., Goodman, Z. D. and Stocker, J. T. (2001) Malignant mesenchymal tumors. In "Tumors of the Liver and Intrahepatic Bile Ducts. Fascicle 31, Atlas of Tumor Pathology, Third Series", ed. by J. Rosai and L. H. Sobin, Armed Forces Institute of Pathology, Washington DC, pp. 281-230.

10. Khanna, C., Khan, J., Nguyen, P., Prehn, J., Caylor, J., Yeung, C., Trepel, J., Meltzer, P. and Helman, L. (2001) Metastasisassociated differences in gene expression in a murine model of osteosarcoma. Cancer Res. 61; 3750-3759.

11. Khanna, C., Wan, X., Bose, S., Cassaday, R., Olomu, O., Mendoza, A., Yeung, C., Gorlick, R., Hewitt, S. M. and Helman, L. J. (2004) The membrane-cytoskeleton linker ezrin is necessary for osteosarcoma metastasis. Nat. Med. 10; 182-186.

12. Köbel, M., Gradhand, E., Zeng, K., Schmitt, W. D., Kriese, K., Lantzsch, T., Wolters, M., Dittmer, J., Strauss, H. G., Thomssen, C. and Hauptmann, S. (2006) Ezrin promotes ovarian carcinoma cell invasion and its retained expression predicts poor prognosis in ovarian carcinoma. Int. J. Gynecol. Pathol. 25; 121-130.

13. Li, Y. R., Akbari, E., Tretiakova, M. S., Hart, J., Akbari, M., Urbanski, S. J. and Gao, Z. H. (2008) Primary hepatic malignant fibrous histiocytoma: clinicopathologic characteristics and prognostic value of ezrin expression. Am. J. Surg. Pathol. 32; $1144-1158$.

14. Mäkitie, T., Carpén, O., Vaheri, A. and Kivelä, T. (2001) Ezrin as a prognostic indicator and its relationship to tumor characteristics in uveal malignant melanoma. Invest. Ophthalmol. Vis. Sci. 42; $2442-2449$ 
15. Mhawech-Fauceglia, P., Dulguerov, P., Beck, A., Bonet, M. and Allal, A. S. (2007) Value of ezrin, maspin and $\mathrm{nm} 23-\mathrm{H} 1$ protein expressions in predicting outcome of patients with head and neck squamous-cell carcinoma treated with radical radiotherapy. $J$. Clin. Pathol. 60; 185-189.

16. Moilanen, J., Lassus, H., Leminen, A., Vaheri, A., Bützow, R. and Carpén, O. (2003) Ezrin immunoreactivity in relation to survival in serous ovarian carcinoma patients. Gynecol. Oncol. 90; 273-281.

17. O'Brien, J. E. and Stout, A. P. (1964) Malignant fibrous xanthomas. Cancer 17; 1445-1455.

18. Rydholm, A. and Syk, I. (1986) Malignant fibrous histiocytoma of soft tissue. Correlation between clinical variables and histologic malignancy grade. Cancer 57; 2323-2324.

19. Torer, N., Kayaselcuk, F., Nursal, T. Z., Yildirim, S., Tarim, A., Nòyan, T. and Karakayali, H. (2007) Adhesion molecules as prognostic markers in pancreatic adenocarcinoma. J. Surg. Oncol. 96; 419-423.

20. Wakamatsu, D., Tsuyama, S., Maezono, R., Kato, K., Ogata, S., Takao, S., Natsugoe, S., Aikou, T. and Murata, F. (2005) Immunohistochemical detection of the cytoskeletal components in gastric parietal cells. Acta Histochem. Cytochem. 38; 331-337.

21. Weiss, S. W. and Enzinger, F. M. (1978) Malignant fibrous histio- cytoma: an analysis of 200 cases. Cancer 41; 2250-2266.

22. Weng, W. H., Ahlén, J., Aström, K., Lui, W. O. and Larsson, C. (2005) Prognostic impact of immunohistochemical expression of ezrin in highly malignant soft tissue sarcomas. Clin. Cancer Res. $11 ; 6198-6204$.

23. Working Group of World Health Organization Classification of Tumors of Soft Tissue and Bone (2002) So-called fibrohistiocytic tumours. In "Pathology and Genetics of Tumors of Soft Tissue and Bone" ed. by C. D. M. Fletcher, K. K. Unni and F. Mertens. International Agency for Research on Cancer Press, Lyon, pp. 109-126.

24. Ye, M. F., Zheng, S., Xu, J. H. and Chen, L. R. (2007) Primary hepatic malignant fibrous histiocytoma: a case report and review of the literature. Histol. Histopathol. 22; 1337-1342.

25. Yu, Y., Khan, J., Khanna, C., Helman, L., Meltzer, P. S. and Merlino, G. (2004) Expression profiling identifies the cytoskeletal organizer ezrin and the developmental homeoprotein Six-1 as key metastatic regulators. Nat. Med. 10; 175-181.

This is an open access article distributed under the Creative Commons Attribution License, which permits unrestricted use, distribution, and reproduction in any medium, provided the original work is properly cited. 